Voix et Images

voixetimages

\title{
Tonalités lointaines (sur l'écriture intimiste de Gabrielle Roy)
}

\section{Jacques Brault}

Volume 14, numéro 3 (42), printemps 1989

Gabrielle Roy

URI : https://id.erudit.org/iderudit/200792ar

DOI : https://doi.org/10.7202/200792ar

Aller au sommaire du numéro

Éditeur(s)

Université du Québec à Montréal

ISSN

0318-9201 (imprimé)

1705-933X (numérique)

Découvrir la revue

Citer cet article

Brault, J. (1989). Tonalités lointaines (sur l'écriture intimiste de Gabrielle Roy).

Voix et Images, 14(3), 387-398. https://doi.org/10.7202/200792ar d'utilisation que vous pouvez consulter en ligne.

https://apropos.erudit.org/fr/usagers/politique-dutilisation/ 


\title{
Tonalités lointaines (sur l'écriture intimiste de Gabrielle Roy)
}

par Jacques Brault, Université de Montréal

\author{
et c' est quelque chose \\ de bien mystérieux que \\ la voix humaine, si \\ personnelle, si prenante \\ souvent, et dont il ne \\ reste rien.
}

José Cabanis

Maintenant, si par hasard on me demandait ce que j'entends par écriture intimiste, je me contenterais de répondre en lisant à haute voix ou plutôt à mivoix une page choisie de Tchekhov, de Katherine Mansfield, de Proust, de Carson McCullers, de Bassani, de beaucoup d'autres, sans oublier Gabrielle Roy. Serais-je pour autant au bout de mes peines? L'intime demande qu'on l'habite longuement et même qu'on risque de s'y enfermer. Il ne se révèle qu'avec lenteur et par chuchotement, dans une espèce de pénombre laiteuse, on ne sait plus si c'est nuit claire ou bien jour de lourds nuages, on mesure mal où commence le moi, où s'achève l'autre. Et quelle voix inconnue murmure à même quelles intonations de silence? On ne sait plus, on ne sait pas qu'on a déjà su. On rêve, on s'étrange, et soudain, à la faveur d'une fatigue, d'un faux mouvement de la main, on sent sous les doigts une fragile peau de papier, on retrouve le fil de sa lecture dont on ignorait qu'on l'avait entreprise.

C'était les mots, on aurait dit, de la maison de chacun, en un jour pareil aux autres, entrecoupés de soupirs et de silences exactement comme dans notre vie où un regard s'échappant par la fenêtre, vers le lointain, en dit tout a coup plus long que les dialogues. Que je trouvai beau, dès que je l'entendis, ce ton du vrai, que ce fût dans la vie ou au théâtre - mais peut-être plus encore au théâtre, qui nous apprend à mieux regarder la vie percée à jour, mise d nu sous nos yeux! Je sentais exprimé comme je n'aurais su le faire moi-même mon propre ennui, mon dépaysement presque constant où que je fusse dans le monde, cette ignorance où l' on est vis-à-vis de soi.

Ces lignes appartiennent à l'un des plus beaux moments de la Détresse et l'enchantement (p. 278-281). Gabrielle Roy assiste, au théâtre des Pitoëff, à une représentation de la Mouette. Son voisin, un jeune inconnu, partage avec elle, du regard, son émotion. Ils échangent quelques mots à l'entracte, puis de retour dans la salle, la magie de Tchekhov les enveloppe à nouveau. Le texte précise: 
Cet étranger près de moi, pendant deux heures et demie, me devint plus proche que presque tous les êtres que javais connus jusque-la. Les voici qui se retrouvent à la sortie, imprégnés encore d'un langage qui les a fait à la fois entrer chacun en soi-même et sortir de soi vers l'autre. Un monologue à deux 'dit l'essentiel, si simple et si loin: C'est ainsi que l'on devrait écrire, ni plus haut, ni plus bas... Le ton juste... il faut peut-être l'avoir cherché toute sa vie pour le trouver à la toute fin... Ils vont comme pour s'effleurer timidement et par quelque voie oblique du geste, mais un passant, figure anonyme du temps qui passe, les écarte l'un de l'autre. Et cet admirable chapitre s'achève en méditation solitaire: On eût dit que Tchékhov, en nous rapprochant, nous avait jeté le même sort qu'd tant de ses personnages, velléitaires, perdus d'indécision, incapables d'aller franchement l'un vers l'autre dans l'élan qui les libérerait.

Ainsi parle l'intimisme littéraire, plus précisément: la tonalité intimiste, dont je veux suggérer ici qu'elle est lointaine.

Henri Michaux affirme que la seule occupation fertile, c'est la pelote inextricable de l'intime. Les dictionnaires, plus prudents et moins incisifs, se bornent à établir qu'en latin intimus est le superlatif d'interior. Mais l'intériorité, si elle connote la profondeur et, par celle-ci, le caché, le secret, le reclus, sa réalité ne reste-t-elle pas sujette à caution? Nombre d'auteurs soutiennent que la profondeur n'est qu'un mirage et que nul n'est doué d'une essence intérieure à son essence individuée. Quant à l'intimité, elle donne lieu à divers malentendus, par exemple chez Alain Finkielkraut: Aujourd' hui, ériger l' intimité en valeur littéraire constitue une régression par rapport aux possibilités de la littérature qui s'exprime le mieux lorsqu' elle a coupé le cordon ombilical avec l'autobiographie. 1

Une première confusion consiste en un amalgame de l'intimité et de l'autobiographie. Que celle-ci fasse ou non l'objet d'un pacte entre l'auteur et le lecteur, peu importe; chaque fois qu'on est forcé de sortir d'un texte littéraire pour le percevoir adéquatement, on le met en doute comme texte littéraire. Il y a plus, infiniment plus. José Cabanis achève son roman, la Bataille de Toulouse, sur ces considérations: Il ne faut faire entrer personne dans certains secrets [...]; cela ne se raconte pas. Mais c'est cela qu'il faut écrire. Et le conseil, exigeant, s'adresse aussi à l'autobiographe. Secondement, l'intimité ne doit pas se confondre avec l'intimisme. L'intériorité (qui a ses professionnels) ou la profondeur (qui a ses aises, ironise Blanchot) ne peuvent nullement à elles seules fonder l'intimisme littéraire, car les grandes images instauratrices de celui-ci appartiennent d'abord au domaine public. Approfondir ou intérioriser ne signifie pas que le sujet langagier s'adonne à la forclusion, bien au contraire, puisqu'il vise à communiquer. Que la forme de cette communication reste en définitive contrariée dans son intention transitive, cela n'empêche pas, le texte littéraire s'auto-désignant, qu'un espace symbolique se déploie, où

1 «Epanchements subjectifs» (propos recueillis par Nicole Czechowski), Autrement, no 81, juin 1986, p. 195. 
l'écrivain et le lecteur sont parties prenantes; l'effusion intimiste reste interne au texte et uniquement au texte.

En somme, la seule caractéristique de l'intimisme qui résiste à l'examen critique des textes, c'est la proximité par quoi l'écriture remodalise la relation du moi et du monde, laquelle je crois constitutive de l'existence et de la connaissance humaines, et de la conscience que l'on peut en avoir. Dans Rue Deschambault, la fin du récit intitulé «Les déserteuses» illustre parfaitement cette propriété fondamentale de l'intimisme. On se souviendra que Christine et sa mère, après leur longue fugue qui les a menées jusqu'au Québec, doivent affronter le ressentiment du père. Les dernières lignes du texte forment à mes yeux l'un des microcosmes de l'œuvre entier de Gabrielle Roy.

Peu à peu nous nous approchions tous de maman pour mieux voir ses yeux qui, avant que ses lèvres les disent, annonçaient les paysages. Car avant de les tirer de son souvenir, son regard les caressait, elle leur souriait, tout en jouant un peu avec le petit collier de perles fausses à son cou. Papa eut une larme à l' æeil, qu'il oublia d' essuyer. Timidement, il demanda d'autres détails: le vieux pommier contre le garage existait-il toujours? Restait-il quelque chose du verger? Et maman les lui donna vrais et touchants. Sur son visage, les souvenirs étaient comme des oiseaux en plein vol. (RD, p. 138)

Mémoire représentative du pays rêvé, précise évocation d'objets familiers, fidèles dans leur absence, tendre humour aussi, baigné de nostalgie, tout ici aménage la proximité qui est le versant diurne de l'intimisme. Le rapprochement affectif diminue l'espace de séparation entre les êtres comme entre l'être et lui-même. La conteuse, inventant à demi (témoin ce petit collier de perles fausses...), a plein les yeux de paroles belles et bonnes et on s'approche pour les voir avant de les entendre. Pourquoi? Parce qu'on veut apprivoiser les mots; les écouter à distance ne suffirait pas à ce qu'ils adviennent au plus près de leur fictive réalité: vrais et touchants. Mais peut-on facilement toucher des oiseaux? Jacques Chardonne là-dessus rumine mélancoliquement: $J$ ai cru aux mots, non aux idées... Mais je voulais te dire quelque chose... - Les mots... Des oiseaux... Ils s'envolent avant qu'on puisse les saisir... ${ }^{2}$ La phrase finale des «Déserteuses» $s$ 'accorde à cette songerie. Elle rapproche aussi la conclusion et l'ouverture du récit alors que des mouettes environnent Christine et sa mère. Voilà donc qu'entre l'éloignement et la proximité s'établit une connivence. C'est aussi cela, l'intimisme: une figure, vitale et fantasmée, qui investit le complexe corps-esprit, le divise, l'unifie, le redivise et le réunifie en un jeu incessant de possession et de dépossession de soi. De soi et du monde, car toujours le Même infuse l'Autre et s'y diffuse - et réciproquement. Telle est l'habitation intimiste du monde comme elle se produit dans les meilleures nouvelles de Katherine Mansfield.

2 Ce matin-là, Chardonne parlait à André Bay, le fils de Camille. La mort était proche. Louis Nucera, le Monde, 13 janvier 1984, p. 16. 
Aucune philosophie savante n'étaye l'espace intimiste où le minime dialogue avec l'immense. Alexandre Chenevert apprivoise son petit-fils un peu sauvage. Mais lorsqu'il sentit cette main logée dans la sienne, si menue et si chaude, Alexandre s'éveilla à une inquiétude aussi vaste que le monde. (AC, p. 146) Qui dit confiance, même blessée d'impossible, dit chaleur rayonnante. Le Médéric de Ces enfants de ma vie, malgré sa jeunesse, ou à cause d'elle, connaît d'expérience le prix de cette chaleur. Dans la Montagne secrète, Pierre ne promet rien à la pauvre Nina, si abandonnée, dans le double sens du mot; il fait mieux. Il saisit la petite main aux ongles courts pour la porter un instant sous sa veste, au chaud (MS, p. 38). «Gagner ma vie», de Rue Deschambault, reprend le leitmotiv: Mais nous, ensemble, nous avions chaud. (RD, p. 293) Ensemble; touchants et touchés.

Ainsi se présente la première figuration de l'espace intimiste. Et son lien médiateur par excellence, n'est-ce pas la demeure, là où se réalise l'immanence au monde et à soi-même? La maison familiale apparaît aux premières lignes de Rue Deschambault, un peu à l'écart de la ville, dans une zone à demi campagnarde. L'état de relatif retirement permet aussi l'habitation songeuse du langage. Parler à voix basse ménage les coins d'ombre où comme au fond des armoires sommeillent les objets de la vie quotidienne. Que l'on pense au Rousseau des Rêveries: il y a l'île (ou le pays), le jardin, la maison, la chambre, le lit, le corps absenté de l'éveil, au plus proche de l'inconscient. Une chambre à soi... La représentation de ce désir, si prégnante, Virginia Woolf lui réserve le sort que l'on sait et Gabrielle Roy dans son autobiographie lui consacre certaines de ses pages les plus ferventes.

L'unique sentiment d'exister à quoi voulait parvenir le Rousseau des Rêveries a besoin d'objectivation concrète, sinon il risque de se dévoyer en autisme. Nid, coquille ou carapace, tente, grotte ou caverne, la demeure donne à l'être intimiste sa seconde peau qui est aussi l'épiderme du monde. Le récit où Christine raconte sa coqueluche monte du fond d'un hamac. La malade bénit sa maladie qui lui est occasion de découvrir la nature environnante. Mon feu, se dit Alexandre Chenevert, aimant le mot. Les objets du monde familier constituent de grands opérateurs d'images intimistes. On le voit en abondance chez la Colette de Naissance du jour. Tous ces objets que touche la main au cours d'une journée, vêtements, savon, tasse, papier, lampe, forment les éléments lexicaux d'une longue phrase quotidienne où se dit à elle-même, par la médiation d'un espace objectal, la demeure du temps. L'intimisme, dont j'évoque ici quelques motifs thématiques, ne se borne pas à l'aménagement d'une patrie psychique autonome qui se contenterait d'être et de se dire son être.

Car il faut préciser que l'intime se conforte en se butant à sa différence. À ce propos, si j'ai accordé une espèce de privilège à la proximité en regard de l'intériorité et de la profondeur, ce n'est pas pour évacuer celles-ci, mais pour marquer fortement que l'intimisme, attitude active et créatrice, se réalise dans le mouvement par quoi l'homogène et l'hétérogène cherchent un contact susceptible de les révéler l'un à l'autre et subséquemment de susciter un tiers composite qui est proprement l'intimisme vécu. De ce point de vue, la relation moi- 
monde n'a en elle-même rien d'intimiste. C'est son aménagement dans l'espace et le temps intimistes qui lui donne son pouvoir intime. En retour, cette relation particularisée, affectée d'une dominante qui la colore et l'unifie, confere au sujet intimiste la faculté d'accueillir, même aux moments imprévus, les signes intimes que le monde nous adresse et dont d'habitude nous n'avons cure.

Bachelard rappelle que la poésie est une métaphysique instantanée. Peutêtre que la durée bergsonnienne a négligé cette expérience temporelle primordiale où l'instant, toujours donné au présent, verticalise son flux horizontal, se dresse en vague, se vaporise et finalement cristallise en étonnement de la conscience. Tels sont les moments de béatitude chez Stendhal qui se désolait de ne pouvoir les prolonger. Forcerait-on les choses et les mots en assimilant à la cristallisation les clignotements de l'entrevision, eux-mêmes cousins des tropismes chers à Nathalie Sarraute? Quoi qu'il en soit, sous savons que la partie se joue aux limites de la perception. Parfois, ces lueurs intermittentes mettent des années à parvenir, en images affectivées, à la conscience expressive. L'œuvre de Gabrielle Roy fourmille de notations, rapides ou détaillées, qui témoignent d'une aptitude à dire la cristallisation d'un instant, lequel, à travers des objets mondains servant de révélateurs comme en photographie, tire le moi de son assoupissement et le milieu environnant de sa banalité pour les accorder l'un à l'autre en une seule et même amitié, avec tous les bonheurs et toutes les douleurs que comporte l'intensité d'un moment de pur intimisme.

Mais l'épreuve de la cristallisation appelle une évacuation du trop-plein émotif. Le besoin de se libérer en l'autre et de libérer l'autre en soi donne ses assises à la société intimiste. J'ai remarqué, après bien des lecteurs, que chez Gabrielle Roy, la joie irruptive cherche tout de suite à se diffuser en partage. Lorsque, dans la Détresse et l'enchantement (p. 285-286), Gabrielle Roy, sur la plate-forme d'un autobus parisien, reçoit une véritable illumination, une vision fugitive, dit le texte, où se superposent le Jardin des Tuileries et les prairies manitobaines, elle a tant de joie au cœur et au corps, comme l'alouette de Bemard de Ventadour, qu'elle se retourne vers les visages qui l'entourent pour s'y refléter, s'y libérer en un reflet qui à son tour serait libérant pour ses voisins. Mais rien ne se produit. La naïve a oublié que le partage intimiste implique la réciprocité.

Deux récits de Rue Deschambault offrent en négatif des images longuement développées du difficile partage intimiste: «Alicia», sur le mode pathologique, «Le jour et la nuit», sur le mode pathétique. Que j'aimerais analyser à loisir ces textes où l'écriture intimiste de Gabrielle Roy ne se fait pas défaut, ou très peu, et qui se maintiennent presque à la hauteur de ce chef-d'œuvre du récit intimiste qu'est De quoi t'ennuies-tu, Éveline? Au-delà de tout ce que signifie de bouleversant pour une petite fille le spectacle de la maladie mentale, de l'absence absolue dans la présence, chez une sœur bien-aimée, "Alicia» pose lumineusement le problème de l'infusion et de la diffusion, complémentaires psychiques et sociaux du partage intimiste. Alicia est à la fois dehors et dedans, et cela sous le même rapport. Elle souffre dans sa chair le principe de contradiction. Et le texte de Gabrielle Roy, plein de pudeur - on le voit au grand 
nombre des indicateurs de doute et de supputation — $s$ 'applique, avec d'infinies précautions, à établir le contact, le tact mutuel. Un seul instant donc, nous fûmes nous-mêmes en Alicia, et Alicia fut elle-même en nous, et nous étions sur une même rive, proches à nous toucher. (RD, p. 179) Un seul instant, oui, comme une pauvre petite ficelle lancée dans un gouffre de solitude.

La figure du père, dans Rue Deschambault, me fascine. Lui aussi, c'est un abîme. Mais en cet homme tombé au fond d'un puits, à Dunrea, où il a trouvé enfin un état de repos complet (RD, p. 159), on peut descendre jusqu'à un certain point. Le père de «Le jour et la nuit» nous est présenté au moment de sa défaite. C'est un errant de la nuit, perclus de peines indicibles, coincé dans sa propre fermeture. Il ne demande presque rien, et quand il demande, sur le tard, c'est avec la certitude qu'il est trop tard: Ne pouvais-tu pas veiller encore une heure avec moi? (RD, p. 275) Ici, le partage intimiste s'annule dans une demande pourtant elle-même intime, mais qui s'acharne à l'échec, car la souffrance de l'arrachement à soi reste plus forte que la dilection de l'être-ensemble. La narratrice conclut sur une phrase terrible de tranquillité: Mon père mourut d l'heure qui lui était la plus cruelle, quand le soleil se lève sur la terre. (RD, p. 277)

L'intimisme n'a pas lieu facilement. Il est rare qu'il trouve demeure à sa modeste démesure. Il exige trop et pas assez. Toute la folle ferveur du monde et la quiète discrétion du moi. Il erre, comme son compère vagabond Éros, à travers les espaces et les temps de nos vies, laissant ici et là des marques subtiles de ses allées et venues. L'écriture intimiste relève ces traces. Comment y arrive-t-elle?

Bien que la tonalité en littérature ne soit pas uniquement intimiste, tout comme est polymorphe et polyvalente la relation instituée par le langage entre le moi et le monde, l'intimisme s'exprime de préférence par la tonalité. Les recherches là-dessus me semblent encore embryonnaires. Restons donc modeste, d'autant plus que même en musique, domaine naturel de la tonalité, les choses ne vont pas toutes seules. Lors d'une belle étude sur Glenn Gould, Michel Schneider observe: L'intonation est difficile d analyser; c'est, si l'on veut, ce qui fait que deux pianistes jouant les mêmes notes avec le même volume, et sur un même piano, produisent des sonorités différentes. ${ }^{3}$ On pourrait tenir des propos à peu près identiques sur la tonalité en peinture. La linguistique, dans l'un de ses chapitres les plus solides, nous a depuis longtemps appris que le ton correspond à la hauteur relative du son de la voix à un moment donné de la châne parlée. C'est donc un élément prosodique et qui dans l'ordre oral appartient au suprasegmental. Par ailleurs, au moyen des accents d'intensité, l'intonation marque des valeurs affectives et régularise l'énonciation. Les difficultés commencent lorsqu'on se met à écrire. Passe-t-on de l'oral au scriptural par simple transposition? Je ne crois pas que l'on gagne beaucoup à se figurer l'intonation

3 Michel Schneider, «Glenn Gould, piano solo», Nouvelle Revue de psychanalyse, no 36 , automne 1987 , p. 26. 
littéraire comme le résultat d'un transvasement de l'oral dans l'écrit. Je pense au contraire que si un texte offre mille et une possibilités significatives, c'est grâce à la tension qui le travaille et à l'effort inventif qu'il déploie pour compenser l'éclatement de la simultanéité orale. Jean Peytard formule ainsi la question inhérente à l'écriture: Comment, dans une successivité linéaire, où tendent à se dissocier et à se disperser les éléments d' un même acte de communication, réinstaurer la présence des relations qui fondent la cohérence de cet acte? ${ }^{4}$

De ce point de vue, la littérature m'apparaît comme une hyjpothèse, non seulement sur le monde et sur le sujet écrivant, mais surtout sur son propre code en tant que celui-ci ressortit à un ensemble de relations internes qui le font, le défont et le refont sans cesse. Car l'écriture du récit (c'est celle qui m'occupe dans cette étude) se doit de combiner du verbal et du non-verbal de telle façon que les fractures résultant de la successivité linéaire ne condamnent pas cette écriture à la banalité de la graphie. Par exemple, la pauvreté informative des indicateurs dit-il, répondit-il, murmure-t-il, les passages systématiques du personnel à l'impersonnel, les interruptions dans la coulée du temps verbal, tous ces facteurs de réduction du sens tendent à diluer le langage littéraire dans le conventionnel. Chaque écrivain s'essaie à contrer cette espèce d'entropie littéraire par un recours paradoxal à l'uniformisation.

Hormis la ponctuation, les artifices typographiques, la versification, la mise en page, quel moyen utiliser pour donner à un texte narratif sa vibration tonale? Claude Roy résume le programme stylistique de Marguerite Duras: Faire surgir le poème dans le tissu de la prose: répétitions et retours d'images - rimes, litanies avec refrains, construction en écho et mouvements de spirale, ellipses et ruptures méditées de ton. ${ }^{5}$ Pas étonnant, avec toute cette quincaillerie, qu'un écrivain par ailleurs prodigieusement doué soit toujours en passe de se caricaturer ou à tout le moins de multiplier les tics d'expression. Chardonne, au contraire, mise sur une limpidité d'élocution alliée à une suggestion finement allitérative: Ce printemps tardif a bondi dans les branches et dans les prés. Déjà, voild le bleu chaud, la lumière qui éblouit, et dans la prairie ensoleillée, je retrouve sous un arbre ce ton oublié de sombre velours ou l'ombre dort. ${ }^{6}$ Quant à Gabrielle Roy, moins baroque que Duras et moins classique que Chardonne, elle possède le don d'évoquer, en un rythme à la fois unifiant et légèrement syncopé, l'attente du moment tonal où fusionnent le bonheur du signifiant et le signifié d'un pathos atténué: Vers la fin du jour, à l' heure qui lui était consolante, quand la lumière faiblissait, que le contour des choses se défaisait, flottait peut-être quelque peu comme dans les rêves, et que la vie paraissait moins dure, mon père se montra. (DE, p. 39)

4 Jean Peytard, «Problèmes de l'écriture du verbal dans le roman contemporain», Nouvelle Critique, décembre 1968, p. 31.

5 Extrait d'un compte rendu de l'Amant, le Nouvel Observateur, 12-18 septembre 1984, p. 67.

6 Cela vient-il de Chimériques ou de Vivre à Madère ou de...? Je ne trouve plus la référence. Avis aux chercheurs. 
Ces deux citations donnent à constater un phénomène étonnant: l'effet tonal entre dans la phrase par la porte d'arrière, si je puis dire, et de là reflue vers l'avant. Voilà une ingénieuse manière de retendre la chaîne écrite et de la tenir, stylistiquement, dans sa dominante tonale. Et les répercussions alors peuvent être innombrables. Pour une, je signale chez Chardonne le contraste simultané où l'ombre s'endort de luisance grâce à l'éveil de la lumière. Chez Gabrielle Roy, on lit l'inverse symétrique: de l'exténuation de la lumière monte une ombre qui gagne en matite. Les nouvelles de Marcel Arland comportent une large gamme de résonnances semblables qui donnent à la narration une allure d'amère jubilation. Un récit de Rue Deschambault, «Petite misère», est typique de l'écriture du livre entier. Si on considère ce texte comme une longue phrase, ce qu'il tente d'être, finalement, on constate qu'il fait alterner les éléments verbaux et les éléments du commentaire, le je narrateur se déplaçant des uns aux autres qu'il parsème d'atténuateurs, souvent les mêmes (peut-être, sans doute, me semble$t-i)$. À ces indices qui sous-tendent et rapprochent les éléments hétérogènes s'ajoutent des accentuations intensives du récit et qui mettent en relief l'expressivité pathétique du personnage paternel. Or, ce qui ressort ici de la surface textuelle, au lieu d'émerger, reflue vers quelque fond supposé. Le pas accablé, le pas découragé du père répercute, au plus bas, sa souffrance traînante, sa douleur pesante. La tonalité dominante de «Petite misère», en contrepoint avec la légèreté des appels lancés vers le grenier, est celle d'une lourdeur de plomb (oui, comme cette tarte à la thubarbe!), d'un chromatisme sombre et terreux. Et le texte suggère de lire en filigrane le secret tout aussi lourd dont la narratrice n'arrive pas à se délivrer, secret sur lequel le texte se replie et coud ses bords, c'est-à-dire le début et la fin dont l'homologie tonale est frappante. Enfin, si je me permets une épreuve par la négative, je m'aperçois que deux paragraphes au moins empêchent le texte d'opérer sa coulée tonale sans heurt. Dans le premier cas, le commentaire se détache et se donne des airs de «pensée morale»; dans le second cas, c'est l'écriture elle-même qui s'embarrasse d'abord dans une inversion superflue et ensuite néglige une inversion qui cette fois s'imposait?

La tonalité en littérature peut, à l'instar de ce qui se passe dans les langues tonales comme le chinois, servir de régulateur à l'écriture qu'elle modalise alors de façon continue comme cela se produit chez Proust et chez Cabanis; elle peut aussi marquer des endroits du texte où une intense émotion affleure en des reflets feutrés, en des inflexions sourdes, qui signent le caractère partiellement intimiste du texte. L'énergie synesthésique de la tonalité se vérifie à l'examen des images impressives qu'elle déploie comme autant de moirages du sens. Le signe symbolique ou representamen de Peirce exerce alors à fond son pouvoir d'attraction. L'écriture tonale invite éminemment à l'association des affects. Les relations internes au scriptural, la sémiotique nous l'aura enseigné, font du langage littéraire un immense carrefour de signes où se distribuent et se redistribuent des rapports immanents, les signes ne se distinguant que par leur différence structurale et fonctionnelle. La littérature puise dans ce réservoir pour 
dresser l'inventaire partiel que constitue chaque cuvre particulière. Mais ce qui empêche la fermeture du sens, toujours menaçante, c'est que le code exige le discursif et donc des relations externes, transcendantes, qui offrent aux signes autant de possibilités de déborder la mise en situation textuelle. Il y a là aussi un risque permanent pour l'écrivain qui ne se contente pas de jouer avec les mots. C'est précisément le rôle de la tonalité que de raccorder l'intérieur et l'extérieur, pour le plus grand bénéfice du sens.

Il existerait ainsi un effet tonal dont la cause principale serait d'ordre sémantique. En disant quelque chose ou même en ne disant presque rien, on dit toujours sa manière d'être au monde. Invoquer un arrière-texte ou un sens caché correspond, je le pense, à une pétition de principe (qui accommode certains chercheurs d'énigmes) et qui appellerait une analyse dont je ferai l'économie. Par contre, là où le sens s'illimite et semble se perdre dans un au-delà de luimême, c'est lorsqu'il s'accomplit comme sens, c'est-à-dire comme mouvement vers l'inextricable des relations internes et externes, dans un va-et-vient par quoi le non-verbal accède tout entier au verbal et où le verbal s'empêche de se fermer sur lui-même. Ici, la tonalité littéraire déploie ses vertus médiatrices avec subtilité, car elle redistribue dans la phrase et dans l'œuvre les effets de sens comme autant de connotations qui déplacent et transhument la lettre du texte. L'implicite se laisse porter par l'explicite qu'en retour il nuance, creusant en lui une différence graduelle qui peut aller du minimal à peine suggéré au choc des contrastes violents. De façon plus technique, André Belleau synthétise mes remarques quand il précise comment un certain non-dit du texte apparaît sur fond d'un signifiant en sourdine, figure verbale d'un énoncé lié d une situation $^{8}$. Ce pourrait être une définition de la tonalité intimiste.

Le «ton retenu» que l'on prête volontiers aux écrivains intimistes n'est pas tellement un indice de pudeur comme celui d'un désir d'allonger, de tenir longuement, je dirais même d'étirer, sémantiquement, l'énoncé. Ce qui, en contrepartie, oblige souvent au tour elliptique, mais pour l'ordinaire, on choisira de «blanchir l'écriture», de la neutraliser, de donner du lisse, de laisser couler, toutes ressources qui justement permettent de condenser les moyens d'expression sans qu'il y paraisse trop et, corrélativement, de laisser du jeu à ce qui est signifié. Si j'infere de la tonalité intimiste qu'elle est lointaine, je crée un paradoxe, puisque l'intimisme, comme mouvement, instaure la proximité. Comment le proche peut-il être loin - et inversement? Dans ce genre de contradiction, une dialectique se tient toujours prête à offrir ses services. Profitons-en. On lit dans «La voix des étangs» l'exacte formulation du problème, qui n'est qu'apparent: Il me semblait que j'étais a la fois dans le grenier et, tout au loin, dans la solitude de l'avenir. (RD, p. 244) Mais un quatrain de la dynastie T'ang9 approfondit et nuance à l'extrême la dialectique du proche et du loin. En voici la traduction littérale:

8 André Belleau, le Romancier fictif, Québec, Presses de l'Université du Québec, 1980 , p. 53.

9 Il s'agit d'un poème de Meng Hao-jan (689-740). 
Sommeil printanier ignorer aube

Tout autour entendre chanter oiseaux

Nuit passée: bruissement de vent, de pluie

Pétales tombés, qui sait combien...

À l'aube de ce matin, le printemps continue à dormir alors qu'un dormeur s'éveille peu à peu. Dans un reste de sommeil, le présent (les oiseaux), le passé (vent et pluie) et l'avenir (le jardin à contempler) se compénètrent sans se confondre. Le plus proche et le plus loin s'appellent en complémentarité certes, mais de telle façon que dure la tension entre eux, et cette tension, indécise, suggestive, offre une grande richesse d'associations. Le poème se répercute dans un passage d'Alexandre Chenevert, j'aime le croire: Soit illusion du sommeil, soit au contraire perception très lucide à la limite du rêve, dans l'instant qu' il s'éveilla, Alexandre Chenevert crut percevoir comme une présence qui se détachait de lui, vague forme qui à regret s'éloignait. (AC, p. 212)

Ainsi, la tonalité intimiste serait lointaine parce que l'intimisme, dans sa dynamique instauratrice d'une existence humanisée, renégocie inlassablement le contrat qui lie le monde et le moi. Dans sa version littéraire, l'intimisme révise et réévalue les modalités langagières du contrat. Car si le prochain et le lointain s'appellent, c'est parce qu'ils se repoussent. Comme disent le bon sens et le bon sieur de La Palice, pour s'approcher, il faut être loin, et pour s'éloigner, il faut être proche. Le langage s'use à l'usage, les associations se perdent, les liens se distendent, le monde s'éloigne dans une altérité brumeuse, le moi se tasse et se massifie. C'est pourquoi on réagit souvent par le rejet quand s'annonce une séparation. Richard Sennett a soigneusement étudié le phénomène, reprenant notre paradoxe sur le plan psycho-social. Les êtres humains ont besoin d'être protégés des autres pour être sociables. Il n'hésite pas à déclarer: Plus les gens sont intimes, plus leurs relations deviennent douloureuses, asociables, fratricides. ${ }^{10}$ Mauriac explorant les Déserts de l'amour avait devancé le sociologue. L'amour-passion n'échappe guère à l'hystérie fusionnelle dans sa phase première - qui souvent est la dernière. Nous sommes faits pour échanger nos différences, en toutes sortes de commerces, avec fantaisie comme avec gravité, non pour nous subjuguer en une fascination où chacun devient le Narcisse de l'autre. L'intimisme correspond à la conscience lucide et ouvrière de l'intime qui, malgré qu'il en ait, comprend la nécessité vitale de s'attacher en se détachant. C'est la leçon de «La voix des étangs», où Christine s'éloigne de son pays familial pour se mettre à l'écoute d'un immense pays sombre (RD, p. 244) d'où lui viendront, elle l'espère, les signes d'un nouveau rapprochement, littéraire celui-là.

Tonaliser l'écriture intimement consiste donc en une double tâche où se signalent l'évitement de la confusion et celui de la séparation, et où le texte, dans sa texture même, gardant toute la tension du verbal et du non-verbal, attire et repousse de manière indécidable. L'écriture intimiste travaille dans l'espace 
minimal d'une fracture psychique où la moindre distance est ressentie comme un éloignement infini. C'est là, en état de malaise et de bien-être, que la tonalité intimiste se pose en hypothèse stylistique. Elle essaie de faire voir et entendre le lointain à proximité, mais pour y parvenir, elle doit s'éloigner du proche. Brisure, blessure, faille, lézarde, cassure, tous ces mots correspondent et suggèrent que les fantasmes intimistes toument comme des flammèches autour d'une béance de ténèbres. Dans le Héron, Bassani nous montre un personnage parti pour la chasse et qui n'en reviendra pas. Le grand oiseau solitaire et taciturne, comme égaré dans les marais d'automne, loin de la chaleur natale, loin de son origine signifiante, reçoit avec gratitude le coup de fusil qui le tue. Une phrase de la Détresse et l'enchantement m'a laissé songeur: De la naissance d̀ la mort, de la mort da la naissance, nous ne cessons, par le souvenir, par le rêve, d'aller comme l'un vers l'autre, d̀ notre propre rencontre, alors que croît en nous la distance. (p. 80)

De la mort à la naissance... Gabrielle Roy n'a pas écrit cela par mégarde. Dans la Route d'Altamont se précise la pensée de l'auteur: À celle qui nous a donné le jour, on donne naissance d notre tour quand, tôt ou tard, nous l'accueillons enfin dans notre moi. Dès lors, elle habite en nous autant que nous avons habité en elle avant de venir au monde. (RA, p. 226-227) Et José Cabanis renchérit, au sujet de son père: Quand il n'a plus été là, nous avons commencé, ensemble, une autre vie. Écrire ainsi commande de quitter les siens et de se quitter, tout comme d'abandonner le monde immédiat à son apparente suffisance. À la mort narcissique de la naissance s'ajoute la mort symbolique de la reconnaissance. On s'exile pour éprouver à fond la nostalgie, pour mettre à l'épreuve ce mal de retour et l'orienter vers un sens nouveau, porteur d'une autonomie, encore douloureuse certes, mais communicable, ou plutôt partageable et donc réconciliatrice du prochain et du lointain. Marcel Arland se modèle sur ce schéma lorsqu'il met en œuvre ses propres tonalités lointaines, métaphores d'un excès rentré ou d'un manque manifesté, ce serait à voir, mais la distinction briserait le vif du sens qui sans doute tient par ce doute même.

Si j'étais mis en demeure de montrer sur un texte où se trouvent au juste les tonalités intimistes, serais-je embarrassé? Après avoir désigné quelques mots, souligné une phrase, repéré un passage, il me viendrait à l'esprit la fine remarque de Montaigne: La parole est moitié à celui qui parle, moitié à celui qui écoute. Un texte de rêverie se compose d'une écriture rêveuse et d'une lecture rêvante. Ainsi en va-t-il pour l'œuvre intimiste. On a raison de prétendre que les affects, particulièrement les affects langagiers, déterminent la lecture des œuvres littéraires. L'Éveline de Gabrielle Roy comprendrait cela d'instinct, ou plutôt à partir d'un savoir intime, elle qui prenait toute chose de langage à la lettre, dans ce qu'elle a de plus précieux et de plus proche, de plus immédiat et de plus menacé. La tentation de l'intimisme littéraire, qu'on l'écrive ou qu'on le lise, c'est, par crainte ou mépris de l'exil de soi, de biffer le monde, la différence, de chuter dans la confession et la confusion, de s'émouvoir au polissage de son moi-bibelot.

La tonalité intimiste reste lointaine et presque éperdue de non-sens parce que rien ne va de soi, ni personne. Lire, comme écrire, entraîne dans l'étran- 
gement et mène au bord du néant, figure muette de notre contingence d'être. Ce qui existe n'a guère de raison d'exister, voilà ce que murmure à la surface du texte l'intime de l'intime, image inversée d'un monde vide et flottant sur le vide. Mais je me mélancolise en une vaine philosophie; laissons cette chère Éveline conclure:

Le front contre la vitre, fouillant du regard le noir de la nuit, un noir comme habité de blancheur, elle découvrit qu'elle éprouvait des sensations nouvelles et mystérieuses, et qu'en cela il y avait une sorte de joie. Et n'était-ce pas singulier que la joie pût venir d'une source aussi impénétrable? (DQE, p. 17)

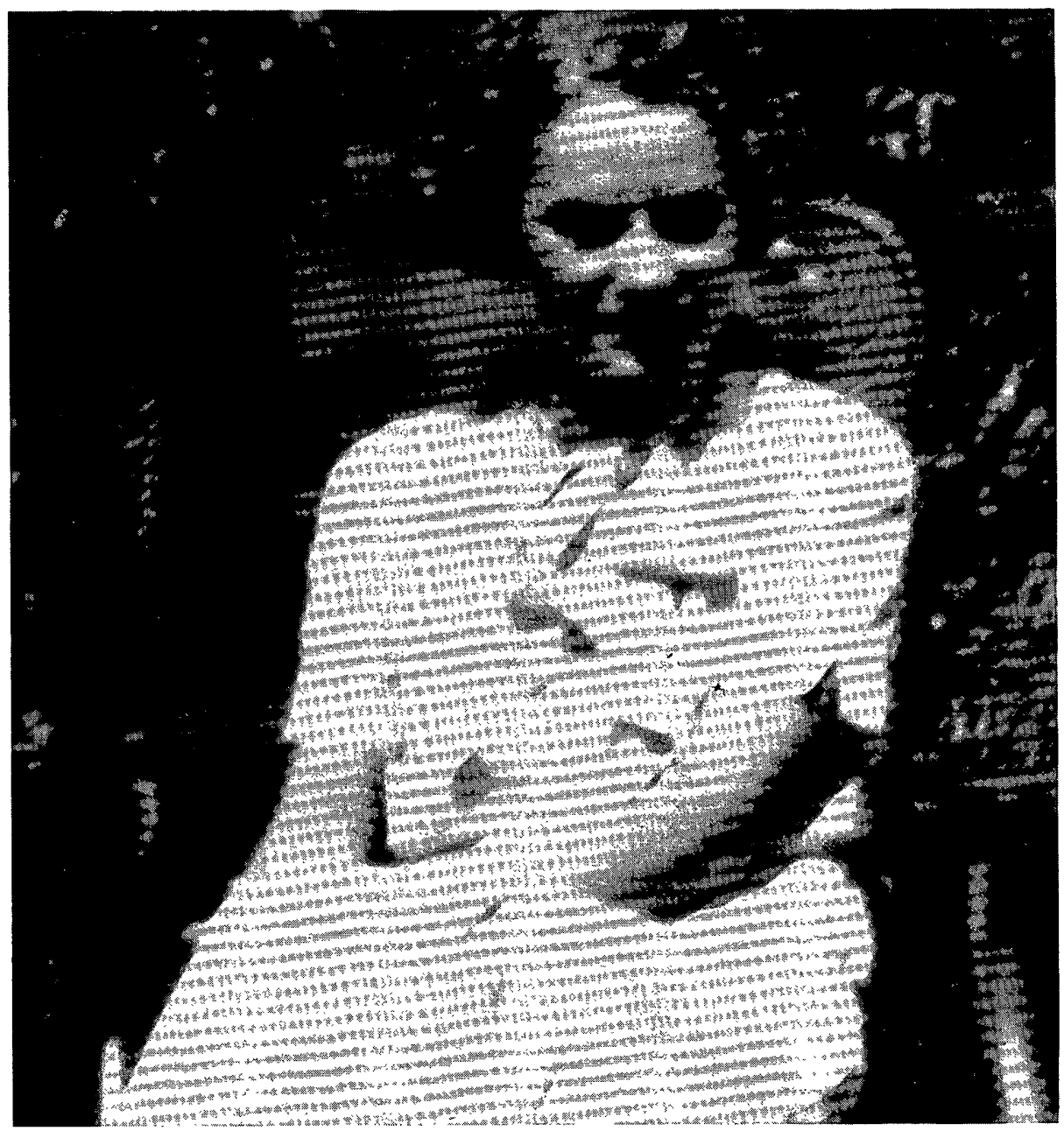

